\title{
Adverse Effects of Kampo Medicines
}

\author{
Yutaka Shimada
}

\begin{abstract}
:
This review summarizes the adverse effects of Kampo medicines. These adverse effects in terms of immunoallergic reactions include interstitial pneumonia, liver injury, allergic cystitis, and drug eruption. Many cases of interstitial pneumonia, liver injury, and allergic cystitis associated with Kampo formulas have been reported to be caused by formulas containing Scutellariae Radix (Scutellaria root, ogon). The known adverse effects linked to overdose of Kampo formulas include pseudoaldosteronism (caused by Glycyrrhizae Radix [licorice, kanzo]), sympathomimetic symptoms (caused by Ephedrae Herba [ephedra, mao]), aconite poisoning (caused by Aconiti Tuber [processed aconite root, bushi and uzu]), and diarrhea (caused by Rhei Rhizoma [rhubarb, daio]). In recent years, mesenteric phlebosclerosis caused by the long-term administration of Gardeniae Fructus (gardenia fruit, sanshishi) has also been reported. It is necessary to consider these potential adverse effects when prescribing Kampo medicines in clinical practice.
\end{abstract}

Key words: Kampo medicines, adverse effect, interstitial pneumonia, liver injury, pseudoaldosteronism, mesenteric phlebosclerosis

(Intern Med Advance Publication)

(DOI: 10.2169/internalmedicine.6292-20)

\section{Introduction}

Traditional Japanese medicines (Kampo medicines) have been considered to be relatively safe with few adverse effects. In general, adverse effects refer to injurious reactions that occur in patients despite the prescription of drugs according to specific criteria. However, there is a traditional understanding that Kampo medicines should be prescribed according to the ideals of Kampo medicine, or the Kampo diagnosis ("sho"), which is considered important for enhancing the efficacy and reducing the risk of adverse effects. That said, Kampo medicines are drugs, and indeed, adverse effects occur even when the medicines are considered to have been prescribed appropriately in terms of the Kampo diagnosis.

Adverse effects, such as pseudoaldosteronism caused by Glycyrrhizae Radix (licorice in English, kanzo in Japanese), sympathomimetic symptoms caused by Ephedrae Herba (ephedra in English, mao in Japanese), aconite poisoning caused by Aconiti Tuber (processed aconite root in English, bushi and uzu in Japanese), and diarrhea caused by Rhei Rhizoma (rhubarb in English, daio in Japanese), are well known. In 1967, Kampo formulations for prescription (i.e. ethical Kampo formulations) were included in the drug price list for the first time, and this list was supplemented with numerous formulations around 1980 in Japan (1). At that time, certain adverse effects caused by Kampo medicines, such as interstitial pneumonia, liver injury, and mesenteric phlebosclerosis, were not widely recognized (Table 1). In addition to academic case reports, the reporting system for adverse drug events established by the Ministry of Health, Labour and Welfare (MHLW) and the Pharmaceuticals and Medical Devices Agency is considered to have contributed significantly to our awareness of the adverse effects associated with Kampo medicines, and in recent years, these adverse effects have been reported increasingly frequently (2).

In this review, the adverse effects of Kampo medicines that should be used with caution are discussed.

\section{Popularization of Kampo Medicines}

\section{Inclusion of Kampo medicines in the drug price list}

In 1967, four ethical Kampo formulations were included for the first time in the drug price list in Japan, and the 
Table 1. Adverse Effects of Kampo Medicines.

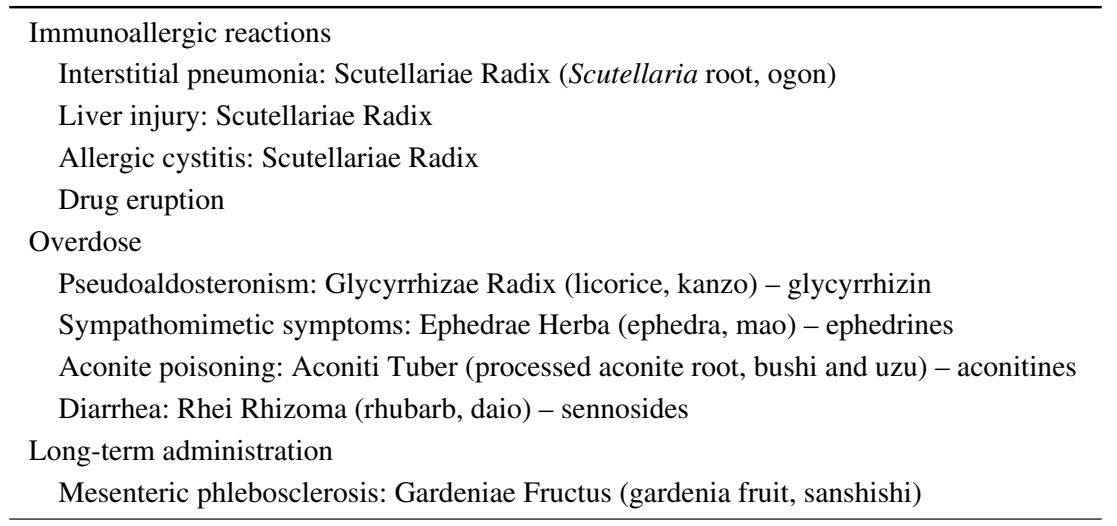

Table 2. Ethical Kampo Formulations Containing Scutellariae Radix (Scutellaria Root, Ogon).

bofutsushosan, daisaikoto, daisaikotokyodaio, gorinsan, hangeshashinto, junchoto, keigairengyoto, nijutsuto, nyoshinsan, ogonto, orengedokuto, otsujito, ryutanshakanto, saibokuto, saikanto, saikokaryukotsuboreito, saikokeishikankyoto, saikokeishito, saikoseikanto, saireito, sammotsuogonto, san'oshashinto, seihaito, seijobofuto, seishinrenshiin, shin'iseihaito, shosaikoto, shosaikotokakikyosekko, unseiin

number of formulations included in this list increased markedly in 1976, 1978, 1981, and 1984. At present, 148 ethical Kampo formulations are available for physicians to prescribe in daily clinical practice under the national health insurance program (1).

When these Kampo formulations were first included in the drug price list, clinical trials had not yet been conducted for them despite usually being conducted for prescription drugs. In addition to ethical Kampo formulations, the prices of approximately 200 types of crude drugs are included in the drug price list, and these agents can be used for the preparation of Kampo prescriptions under the national health insurance program.

\section{Popularization of ethical Kampo formulations}

According to the Japan Kampo Medicines Manufacturers Association (JKMA) (3), the annual production value of ethical Kampo formulations was 2.652 billion yen in 1976 . Following the inclusion of a large number of ethical Kampo formulations in the drug price list around 1980, this value increased markedly, reaching 154.196 billion yen in 1992 . However, due to the adverse effects of shosaikoto, which will be described later, the value decreased to 84.112 billion yen in 2000 and thereafter increased again to 151.396 billion yen in 2018 .

As a side note, according to a survey conducted by the JKMA in 2011, 89\% of physicians reported prescribing Kampo medicines to their patients $(1,4)$.

\section{Adverse Effects Due to Immunoallergic Reactions}

Since around 1990, it has been known that Kampo medicines may cause adverse effects, such as interstitial pneumonia and liver injury. Many of these adverse effects have been reported to be caused by Kampo formulas containing Scutellariae Radix (Scutellaria root in English, ogon in Japanese) (5). Although the detailed mechanisms underlying the adverse effects of Kampo medicines have not been clarified, it is assumed that immunoallergic reactions are involved. Table 2 shows the ethical Kampo formulations containing Scutellaria root.

\section{Lung injury}

Drug-induced lung injury (interstitial pneumonia) caused by shosaikoto was first reported in 1989 (6), and similar reports continued in the 1990s. The Ministry of Health and Welfare issued Dear Healthcare Professional Letters of Emergent Safety Communications (Yellow Letter) for shosaikoto in 1996 (7). Thereafter, interstitial pneumonia induced by Kampo formulas other than shosaikoto were also reported, and in many cases, the condition was caused by Kampo formulas containing Scutellaria root $(5,8)$. The MHLW publishes data on adverse effect reports on its website. Based on the author's analysis of these data, approximately $70 \%$ of Kampo formulas that were suspected to have caused adverse effects related to lung injury were those containing Scutellaria root, such as saireito, bofutsushosan, otujito, hangeshashinto, shosaikoto, seishinrenshiin, and saikokaryukotsuboreito (2). According to a report on drug- 
induced interstitial lung injury from 2004 to 2009 that incorporated investigations by the Japanese Respiratory Society, $233(3.1 \%)$ of the 7,598 cases were attributable to Kampo medicines (9). Furthermore, the incidence of interstitial pneumonia caused by Kampo formulas containing Scutellaria root was $3(0.27 \%)$ among 1,111 patients (10).

In some of the package inserts of ethical Kampo formulations, interstitial pneumonia is described as a serious adverse effect, although the expression somewhat differs across package inserts: "If symptoms such as fever, cough, dyspnea, and abnormal lung sounds (crepitation) are noted, the medicine should be discontinued, and chest X-ray and other examinations should be promptly performed, and appropriate treatments such as corticosteroids should be started. In addition, caution should be exercised if symptoms such as fever, cough, and dyspnea develop, then the medicine should be discontinued and physicians should be consulted immediately." Many of these Kampo formulas contain Scutellaria root. As a warning, the package insert of shosaikoto has almost the same description as the above-mentioned serious adverse effects, and the medicine is contraindicated in patients receiving interferon preparations, patients with cirrhosis or liver cancer, and patients with chronic hepatitis with a platelet count of $\leq 100,000 / \mathrm{mm}^{3}$.

\section{Liver injury}

There are many reports of Scutellaria root-containing Kampo formulas as causes of drug-induced liver injury as well as lung injury (5). According to the author's analysis based on the data of adverse effects reported by the MHLW, approximately $70 \%$ of Kampo formulas suspected to have caused adverse effects related to liver injury were those containing Scutellaria root, such as bofutsushosan, saireito, saikokaryukotsuboreito, orengedokuto, hangeshashinto, otsujito, saikokeishikankyoto, saibokuto, and shin'iseihaito (2). According to an analysis of 1,676 cases of drug-induced liver injury between 1997 and 2006 conducted by the Japan Society of Hepatology, causal drugs were narrowed down to a single medicine in 879 cases, and the result was a Kampo formula in $7.1 \%$ of the cases (11). Regarding the incidence of Kampo formula-induced liver injury, a study reported that $13(1.0 \%)$ of 1,328 patients who received Kampo formulas containing Scutellaria root developed liver injury (12).

Some package inserts of ethical Kampo formulations mention the following, although the expression may slightly differ across package inserts: "Liver dysfunction and jaundice may develop accompanied by a marked increase in AST (GOT), ALT (GPT), Al-P, and $\gamma$-GTP levels and those of others; therefore, patients should be observed carefully, and the medicine should be discontinued and appropriate treatment should be administered if any abnormalities are observed." Many-but not all-of these Kampo formulas contain Scutellaria roots. The package insert of shosaikoto states the following as important basic points of caution: "Be careful of a change in the platelet count of a patient with chronic hepatitis during the administration of the medi- cine for the treatment of liver dysfunction, and discontinue the medicine if the platelet count decreases." Furthermore, in the package insert of saireito, "fulminant hepatitis" is also described as a serious adverse effect.

\section{Allergic cystitis}

Allergic cystitis (interstitial cystitis) caused by Kampo medicines has been reported, albeit rarely. According to the author's analysis based on the data of adverse effects reported by the MHLW, in most instances, the condition was caused by Kampo formulas containing Scutellaria root (2). In the package inserts of shosaikoto, saikokeishito, saibokuto, and saireito, it is mentioned that cystitis-like symptoms, such as frequent urination, micturition pain, hematuria, and feeling of residual urine, may develop as adverse effects.

\section{Drug eruption}

There are reports of drug eruptions, including StevensJohnson syndrome, caused by Kampo medicines. According to the author's analysis based on the data of adverse effects reported by the MHLW, Kampo formulas containing ephedra accounted for about $35 \%$ of the reports (2).

\section{Adverse Effects Due to Overdose}

Although there are individual differences in reactivity, adverse effects caused by Kampo medicine overdose have been reported. These adverse effects have been recognized since before 1980, when a considerable number of ethical Kampo formulations were included in the drug price list.

\section{Pseudoaldosteronism caused by licorice}

Glycyrrhizin contained in licorice causes pseudoaldosteronism, i.e. edema, hypertension, hypokalemia, and further persistent hypokalemia, which may result in muscle injuries, such as myopathy and rhabdomyolysis, and cardiac problems, such as arrhythmia and heart failure. According to the description on the package insert of the ethical Kampo formulations containing licorice, products containing $\geq 2.5 \mathrm{~g}$ of licorice per day are contraindicated for patients with aldosteronism, myopathy, and hypokalemia because they may worsen these diseases or symptoms. In addition, caution should be exercised when using licorice-containing products, glycyrrhizic acid and its saline-containing products, loop diuretics (furosemide, ethacrynic acid), and thiazide diuretics (trichlormethiazide) in combination. Furthermore, the following important basic points of caution are included for these products: "Since the medicine contains licorice, closely monitor serum potassium levels and blood pressure, and discontinue the medicine if any abnormalities are observed. In addition, be careful about overlapping of the crude drug when using other Kampo products concomitantly."

Among the 148 ethical Kampo formulations available, 109 contain licorice (13). The most commonly prescribed 
ethical Kampo formulations for which pseudoaldosteronism has been reported as an adverse effect are shakuyakukanzoto and yokukansan (2). The reason for this is considered to be the high licorice content and frequent administration of the medicines. Shakuyakukanzoto has a high licorice content and is often prescribed for muscle cramps and painful diseases. Yokukansan has a relatively low licorice content, but its prescription for the treatment of behavioral and psychological symptoms of dementia has increased in recent years, leading to an increase in reports of pseudoaldosteronism.

Regarding the mechanism underlying pseudoaldosteronism, the ingested glycyrrhizin is believed to be converted to glycyrrhetinic acid via the involvement of intestinal bacteria. Since glycyrrhetinic acid has a strong inhibitory effect against 11ß-hydroxysteroid dehydrogenase II, which degrades cortisol to cortisone in the cytoplasm of renal tubular cells, the increased cortisol acts on the mineralocorticoid receptors in renal tubules and promotes sodium reabsorption and potassium excretion (13). A recent study reported that sulfotransferase $2 \mathrm{~A} 1$ is a key enzyme involved in licoriceinduced pseudoaldosteronism; furthermore, a novel glycyrrhizin metabolite was identified as a potential biomarker (14).

\section{Sympathomimetic symptoms caused by ephedra}

Sympathomimetic symptoms, such as tachycardia, palpitation, increased blood pressure, hyperhidrosis, dysuria, and agitation, are caused by ephedrines contained in ephedra. The package inserts of ethical Kampo formulations containing ephedra contain the following information: "Caution should be exercised in the form of measures such as dose reduction when using products containing ephedra, ephedrines, monoamine oxidase (MAO) inhibitors, thyroid preparations (thyroxine, liothyronine), catecholamine preparations (adrenaline, isoprenaline), and xanthine preparations (theophylline, diprophylline) in combination, because the medicine will enhance sympathomimetic effects and may be more likely to cause conditions such as insomnia, hyperhidrosis, tachycardia, palpitation, general weakness, and mental agitation."

\section{Aconite poisoning caused by processed aconite root}

Aconitines contained in processed aconite root may cause adverse effects of aconite poisoning, such as neuroparalysis. Bushi and uzu are post-processing products of tuberous aconite roots and used as crude drugs in Kampo medicine. Early symptoms of aconite poisoning include numbness of the lip or tongue and palpitation, followed by nausea, vomiting, and abdominal pain, as well as terminal illness symptoms, such as arrhythmia and respiratory paralysis. Although some ethical Kampo formulations contain processed aconite root, there are few reports of actual related adverse effects (2). However, caution should be exercised when administering processed aconite root powder or bushi and uzu as crude drugs, as adverse effects tend to occur at high doses. In the case of crude drugs, the decoction time is also important.

\section{Diarrhea caused by rhubarb}

Sennosides contained in rhubarb may cause diarrhea. According to the package inserts of ethical Kampo formulations containing rhubarb, cautious administration is recommended for patients with diarrhea or loose stools because the symptoms may worsen.

\section{Adverse Effects Due to Long-term Administration}

\section{Mesenteric phlebosclerosis caused by gardenia fruit}

Mesenteric phlebosclerosis is characterized by a chronic ischemic large bowel lesion caused by impaired perfusion due to mesenteric vein sclerosis and was first reported in Japan in the 1990s. Asian cases, mainly Japanese ones, have been reported, and there have been many cases reported among individuals using Kampo medicines, especially formulas containing Gardeniae Fructus (gardenia fruit in English, sanshishi in Japanese), for a long period of time (several years to several decades) (15). Nagata et al. (16) examined the relationship between the dosage of gardenia fruit and mesenteric phlebosclerosis and reported that the patients had a history of taking the formulation for at least 4 years in the case of onset and whose cumulative dosage was $\geq$ $5,000 \mathrm{~g}$.

The underlying cause of mesenteric phlebosclerosis associated with formulas containing gardenia fruit is the geniposide constituent, which is hydrolyzed by bacteria in the large intestine. The genipin generated is absorbed from the large intestine and reaches the liver through the mesenteric vein, where it then reacts with amino acids and proteins and leads to the formation of blue-colored matter. The reactions also cause fibrous thickening and calcification of the mesenteric vein wall, congestion of blood flow, edema, fibrosis, calcification, and stenosis of the intestinal wall. The major symptoms of mesenteric phlebosclerosis include abdominal pain (on the right side), diarrhea, nausea, and vomiting; however, there are asymptomatic cases as well, including those with a positive occult blood test. In addition, ileus may be present in severe cases (15).

In 2013, the package inserts of the ethical Kampo formulations orengedokuto, kamishoyosan, and shin'iseihaito listed mesenteric phlebosclerosis as a serious adverse effect. In 2014, mesenteric phlebosclerosis was reported to be caused by inchinkoto in the package insert of the formulation. In 2018, it was also described as being caused by all gardenia fruit-containing ethical Kampo formulations and the gardenia fruit itself as a crude drug, with the package inserts of the formulations providing the following information as important basic points of caution: "Long-term administration of gardenia fruit-containing products (often over 5 years) has been reported to cause mesenteric phlebosclero- 


\title{
Table 3. Ethical Kampo Formulations Containing Gardeniae Fructus (Gardenia Fruit, Sanshishi).
}

\author{
bofutsushosan, gorinsan, inchinkoto, kamikihito, kamishoyosan, keigairengyoto, \\ orengedokuto, ryutanshakanto, saikoseikanto, seihaito, seijobofuto, shin'iseihaito, \\ shishihakuhito, unseiin
}

sis, resulting in color abnormalities, edema, erosions, ulcers, and stenosis in the large intestine. In case of long-term use of gardenia fruit-containing Kampo formulations, it is desirable to perform CT, colonoscopy, and other tests regularly." Furthermore, the package inserts highlight the following as a serious adverse effect: "Long-term use of the medicine may cause mesenteric vein sclerosis. If symptoms such as abdominal pain, diarrhea, constipation, and abdominal distention develop repeatedly, or if the fecal occult blood test is positive, the medicine should be discontinued and CT, colonoscopy, and other tests should be performed, and also appropriate treatments should be administered. In some cases, colectomy has been reported to be necessary." Table 3 shows the ethical Kampo formulations containing gardenia fruit.

\section{Adverse Effects Due to Toxicity}

\section{Aristolochic acid nephropathy}

Aristolochic acid nephropathy is a rapidly progressive interstitial nephritis leading to end-stage renal disease and urothelial malignancy. It was first reported in 1993 from Belgium in patients who had ingested slimming pills containing powdered root extracts of Chinese herbs (17) and was initially called Chinese herb nephropathy. It occurred due to the substitution of Stephania tetrandra with Aristolochia fangchi rich in aristolochic acid, as both herbs share the same common name in Pinyin (Han Fang Ji and Guang Fang Ji), and they can be used interchangeably in traditional Chinese medicine irrespective of their botanical classification. Since the index cases, several cases of aristolochic acid nephropathy have been reported worldwide (18).

The crude drug Guang Fang Ji (koboi in Japanese) containing aristolochic acid was reportedly mistakenly imported to Japan as Han Fang Ji (kanboi in Japanese) and used as Sinomenium stem and rhizome (boi in Japanese, listed in the Japanese Pharmacopoeia). The occurrence of aristolochic acid nephropathy in Japan may be associated with this drug. Such mix-ups may occur due to the similarities in the forms and names of these two crude drugs. Such cases have been associated with the import of traditional Chinese medicines or traditional Chinese medicinal products from China and Taiwan, as well as decoctions and health foods similar to these medicines, to Japan for personal use. The crude drugs and Kampo products approved as drugs in Japan do not contain aristolochic acid. The MHLW revised the risk of renal disorders caused by aristolochic acid in the Pharmaceuti- cals and Medical Devices Safety Information in 2000 and 2004 (19), because such cases have been continuously reported in Japan as well as other countries.

\section{Recommendations for Monitoring Adverse} Effects Caused by Kampo Medicines

As Kampo medicines are also drugs, any guidelines or official consensus statements for the diagnosis and treatment of adverse effects, such as drug-induced lung injuries (9), should be followed. As mentioned earlier, various precautions are described in the package inserts of ethical Kampo formulations, and they should be adhered to.

Personal recommendations for reducing adverse effects caused by Kampo formulas especially those containing Scutellaria root, licorice, and gardenia fruit (Table 4), are described below.

\section{Recommendations when prescribing Kampo formu- las containing Scutellaria root}

As mentioned earlier, approximately $70 \%$ of lung or liver injuries caused by Kampo medicines are associated with formulas containing Scutellaria root (2). Physicians should be aware of whether or not a patient has suffered from adverse effects, such as liver injury, lung injury, and allergic cystitis, due to Kampo formulas containing Scutellaria root in the past, and if they have, they should not be prescribed such formulas. When physicians prescribe Kampo formulas containing Scutellaria root to patients for the first time, blood tests, including liver function tests (AST, ALT, AL-P, $\gamma$-GTP, and T-Bil), and chest X-ray are recommended to be performed before prescribing these agents.

Regarding the duration between the start of Kampo medicine intake and the onset of interstitial pneumonia, the cumulative incidence rate until 2 months was reported to be approximately $80 \%$ (5), and that until 3 months was approximately $90 \%$ (8). Therefore, attention should be paid to symptoms such as a fever, cough, and dyspnea, especially within two to three months after beginning the administration of Kampo formulas containing Scutellaria root. A previous study showed that the white blood cell count, Creactive protein, lactate dehydrogenase, and Krebs von den Lungen-6 levels were frequently increased in the blood of patients with Kampo medicine-induced pneumonitis, and most of the patients presented with bilateral ground-glass attenuations on chest computed tomography (CT) or highresolution CT (HRCT) (8).

In contrast, in the case of liver injury, the duration between the start of Kampo medicine intake and the onset 
Table 4. Recommendations to Monitor Adverse Effects Caused by Kampo Medicines.

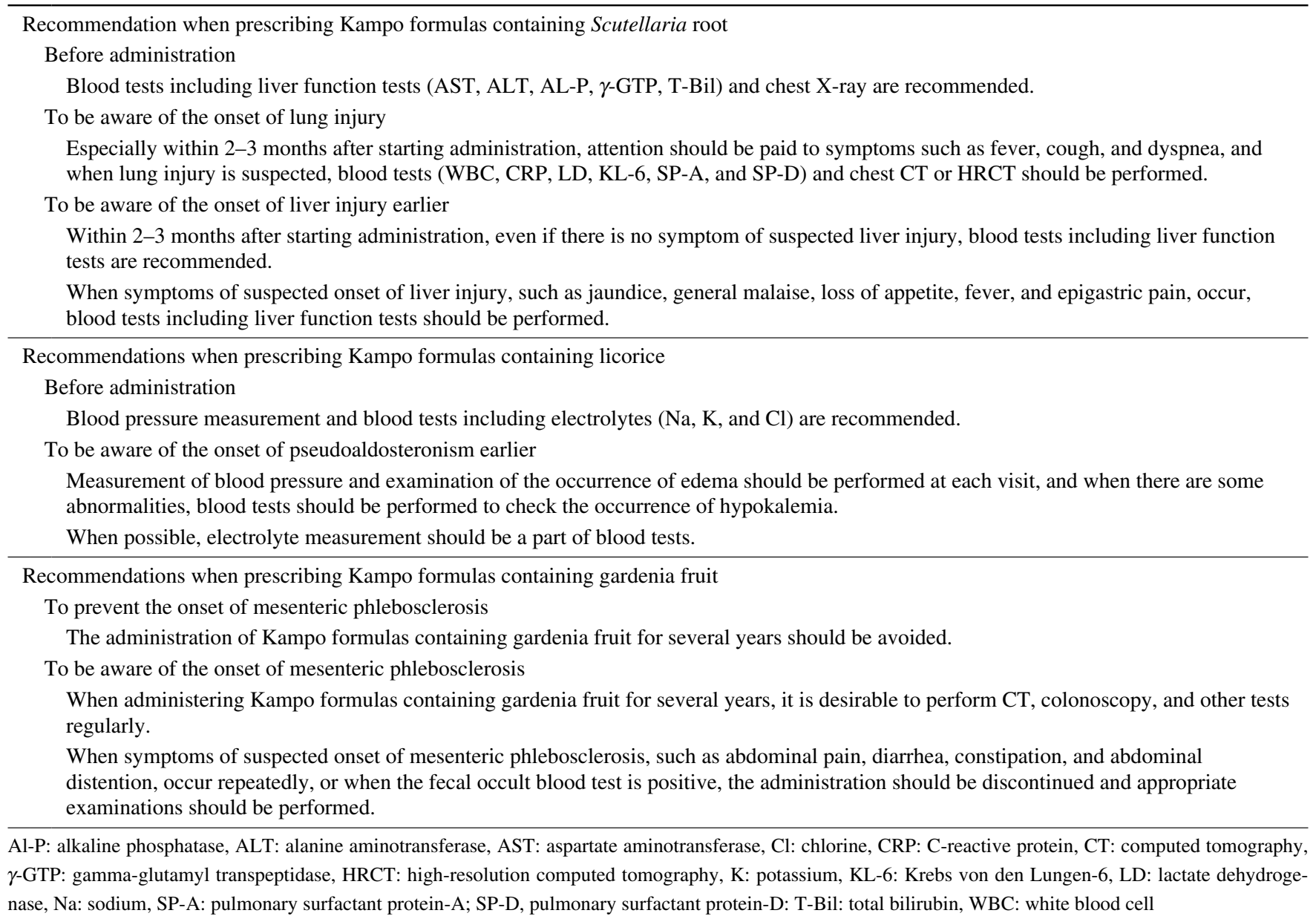

ranged from several days to one year (5). Except for jaundice, most of the symptoms of liver injury caused by Kampo medicines are non-specific, such as general malaise, appetite loss, a fever, and epigastric pain, and it is not uncommon for liver function abnormalities to only be noted at medical checkups (5). This might be one of the reasons why the diagnosis of liver injury tends to be delayed compared with lung injury. Blood tests, including liver function tests, within two to three months after prescribing Kampo formulas containing Scutellaria root are recommended even if there are no symptoms suggestive of liver injury. Of course, when symptoms suggesting the onset of liver injury, such as jaundice, general malaise, appetite loss, a fever, and epigastric pain, occur, the liver function should be examined.

\section{Recommendations when prescribing Kampo formu- las containing licorice}

Blood pressure measurement and blood tests, including evaluations of electrolytes $(\mathrm{Na}, \mathrm{K}$, and $\mathrm{Cl})$, are recommended before prescribing formulas containing licorice, especially at a relatively high content. The measurement of the blood pressure and examinations for edema should be performed at each visit, and when abnormalities are noted, blood tests should be performed to check for the occurrence of hypokalemia. In addition, when possible, electrolyte measurement should be performed along with blood tests. If a patient has hypokalemia, licorice-containing formulas should be discontinued, and appropriate treatments should be administered. As mentioned earlier, the progression of hypokalemia can lead to muscle injuries, such as myopathy and rhabdomyolysis, and cardiac problems, such as arrhythmia and heart failure, which must be prevented before their development.

\section{Recommendations when prescribing Kampo formu- las containing gardenia fruit}

As Kampo formulas containing gardenia fruit are associated with mesenteric phlebosclerosis development, their administration for periods of several years should be avoided. When they are administered for such long periods, as stated in the package inserts, tests such as CT and colonoscopy should be performed regularly. Furthermore, if symptoms suggesting the onset of mesenteric phlebosclerosis, such as abdominal pain, diarrhea, constipation, and abdominal distention, occur repeatedly or if the fecal occult blood test is positive, the administration of formulations should be discontinued, and appropriate examinations and treatments should be performed. 


\section{Conclusions}

In addition to ethical Kampo formulations, there are many over-the-counter (OTC) Kampo formulations, some of which are sold under different names. For example, bofutsushosan, which has been widely used to manage obesity and metabolic syndrome in recent years, has been renamed and sold by many pharmaceutical companies. Since this formula contains crude drugs such as Scutellaria root, gardenia fruit, licorice, and ephedra, it is a Kampo formula that requires attention, especially owing to the possible adverse effects. Indeed, there are reports of adverse effects caused by OTC Kampo formulations.

Recently, there has been concern about polypharmacy in Kampo medicines (20). In recent years, the prescription of Kampo medicines has become popular among physicians, and there are cases in which multiple Kampo formulations are prescribed for a single patient by multiple clinical departments or medical institutions. Since Kampo formulas are a mixture of multiple crude drugs, in such cases, this can result in an overdose of certain ingredients. The most common case involves the use of multiple licorice-containing formulas, which increases the risk of developing pseudoaldosteronism. In addition, the author experienced a case of a patient who was undergoing Kampo treatment in the author's department and repeatedly developed drug-induced liver injury caused by Scutellaria root-containing formulations prescribed by other medical institutions (21). When prescribing Kampo medicines, it is important to keep in mind the constituent crude drugs and adverse effects of Kampo formulas and to obtain information on the status of the use of Kampo medicines, including OTC Kampo formulations, and the history of adverse effects of Kampo medicines.

\section{The author states that he has no Conflict of Interest (COI).}

\section{References}

1. Japan Kampo Medicines Manufacturers Association. [Kampo future vision 2040] [Internet]. 2018 Jul 20 [cited 2020 Aug 31]. Available from: https://www.nikkankyo.org/action/pdf/vision2040.p df (in Japanese).

2. Shimada Y, Fujimoto M, Nogami T, Watari H. Adverse events associated with ethical Kampo formulations: analysis of the domestic adverse-event data reports of the Ministry of Health, Labor, and Welfare in Japan. Evid Based Complement Alternat Med 1643804, 2019.

3. Japan Kampo Medicines Manufacturers Association. [Production dynamics of Kampo products] [Internet]. 2019 Sep 20 [cited 2020 Aug 31]. Available from: https://www.nikkankyo.org/serv/moveme nt/h30/all.pdf

4. Japan Kampo Medicines Manufacturers Association. [Actual condition survey of Kampo prescriptions] [Internet]. 2011 Oct 18 [cited 2020 Aug 31]. Available from: https://www.nikkankyo.org/se rv/pdf/jittaichousa2011.pdf (in Japanese).

5. Terada M, Kitazawa H, Kawakami J, Adachi I. Pharmacoepidemi- ology of interstitial pneumonia and liver dysfunction associated with Kampo medicine. Iryo Yakugaku (Japanese Journal of Pharmaceutical Health Care and Sciences) 28: 425-434, 2002 (in Japanese, Abstract in English).

6. Tsukiyama K, Tasaka Y, Nakajima M, et al. A case of pneumonitis due to sho-saiko-to. Nihon Kyobu Shikkan Gakkai Zasshi (Japanese Journal of Thoracic Diseases) 27: 1556-1561, 1989 (in Japanese, Abstract in English).

7. Serious adverse effect "interstitial pneumonia" caused by administration of shosaikoto: from Pharmaceuticals Safety Information No. 137 (May 1996). Nihon Toyo Igaku Zasshi (Japanese Journal of Oriental Medicine) 47: 81-83, 1996 (in Japanese).

8. Enomoto Y, Nakamura Y, Enomoto N, Fujisawa T, Inui N, Suda T. Japanese herbal medicine-induced pneumonitis: a review of 73 patients. Respir Investig 55: 138-144, 2017.

9. Kubo K, Azuma A, Kanazawa M, et al. Consensus statement for the diagnosis and treatment of drug-induced lung injuries. Respir Investig 51: 260-277, 2013.

10. Nogami T, Fujimoto M, Shimada Y, et al. Incidence of kampo medicine-induced interstitial pneumonia: 10 year retrospective study at a university hospital kampo medicine department. Tradit Kampo Med 6: 26-31, 2019.

11. Takikawa H, Murata Y, Horiike N, Fukui H, Onji M. Druginduced liver injury in Japan: an analysis of 1676 cases between 1997 and 2006. Hepatol Res 39: 427-431, 2009.

12. Itoh $T$, Sugao $M$, Chijiwa $T$, et al. Clinical characteristics of side effects induced by administration of Glycyrrhizae Radix and Scutellaria Radix under the therapy based on kampo diagnosis in our hospital. Nihon Toyo Igaku Zasshi (Kampo Medicine) 61: 299-307, 2010 (in Japanese, Abstract in English).

13. Makino T. 3-Monoglucuronyl glycyrrhretinic acid is a possible marker compound related to licorice-induced pseudoaldosteronism. Biol Pharm Bull 37: 898-902, 2014.

14. Takahashi K, Yoshino T, Maki Y, et al. Identification of glycyrrhizin metabolites in humans and of a potential biomarker of liquorice-induced pseudoaldosteronism: a multi-centre crosssectional study. Arch Toxicol 93: 3111-3119, 2019.

15. Ohtsu K, Matsui T, Nishimura T, et al. Association between mesenteric phlebosclerosis and Chinese herbal medicine intake. Nihon Shokakibyo Gakkai Zasshi (Journal of Japanese Society of Gastroenterology) 111: 61-68, 2014 (in Japanese, Abstract in English).

16. Nagata $Y$, Watanabe $T$, Nagasaka $K$, et al. Total dosage of gardenia fruit used by patients with mesenteric phlebosclerosis. BMC Complement Altern Med 16: 207, 2016.

17. Vanherweghem JL, Depierreux M, Tielemans C, et al. Rapidly progressive interstitial renal fibrosis in young women: association with slimming regimen including Chinese herbs. Lancet 341: 387391, 1993.

18. Debelle FD, Vanherweghem JL, Nortier JL. Aristolochic acid nephropathy: a worldwide problem. Kidney Int 74: 158-169, 2008.

19. Pharmaceuticals and Medical Devices Safety Information No. 200 April 2004 [Internet]. [cited 2020 Nov 18]. Available from: https:// www.pmda.go.jp/files/000153652.pdf

20. Chino A, Tsuji M, Yagi A, Terasawa K. Polypharmacy caused by Kampo prescriptions. Nihon Toyo Igaku Zasshi (Kampo Medicine) 70: 72-76, 2019 (in Japanese, Abstract in English).

21. Shimada Y, Fujimoto M, Nogami T, et al. Recurrent drug-induced liver injury caused by the incidental readministration of a Kampo formula containing Scutellariae Radix. Intern Med 57: 1733-1740, 2018.

The Internal Medicine is an Open Access journal distributed under the Creative Commons Attribution-NonCommercial-NoDerivatives 4.0 International License. To view the details of this license, please visit (https://creativecommons.org/licenses/ by-nc-nd/4.0/). 
(C) The Japanese Society of Internal Medicine Intern Med Advance Publication 